\title{
Can 9q34.2 rs633862 polymorphism predicate survival in epithelial ovarian cancer?
}

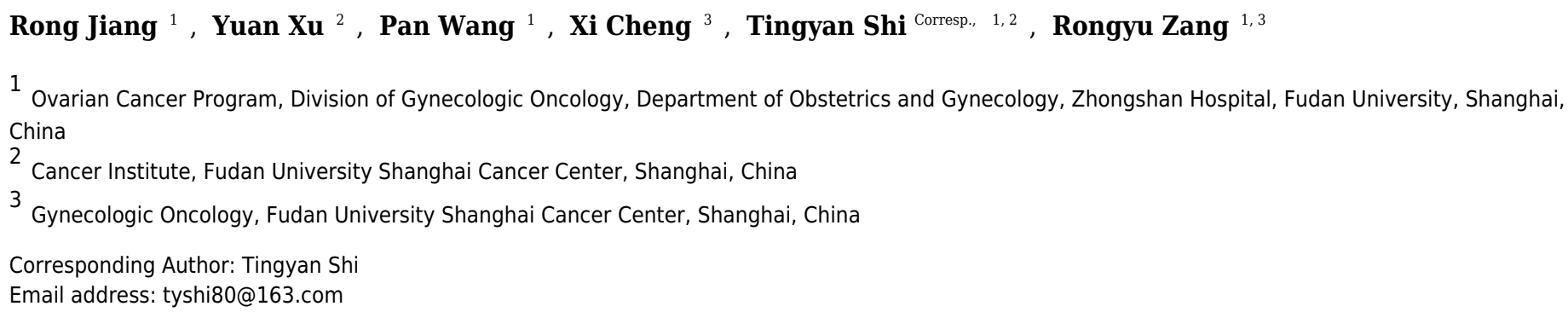

Objective Our previous genome-wide association study (GWAS) has identified that the $A B O$ rs633862 variant in Chromosome 9q34.2 was associated with the risk of epithelial ovarian cancer (EOC) in Chinese Han women. The aim of present study was to evaluate its prognostic effect on EOC. Methods A total of 669 EOC patients were enrolled for the genotyping of rs633862 variant in 9q34.2. We used Kaplan-Meier survival curves, univariate and multivariate Cox proportional hazard models to evaluate the association of rs633862 with overall survival (OS) in EOC patients. Results We found that rs633862 variant AG/GG genotypes were significantly associated with a longer OS by using univariate Cox proportional hazards regression analysis, compared with the rs633862 AA genotype $(\mathrm{HR}=0.69,95 \% \mathrm{Cl} 0.49-0.98, p=0.035)$, albeit with a boardline significance in the multivariate analysis. Similar findings were observed in the subgroup of high-grade serous ovarian carcinoma. Further expression quantitative trait loci (eQTL) analysis indicated that the rs633862 AA genotype was associated with an increased level of $A B O$ mRNA expression ( $p=1.8 \times 10^{-11}$ ). Conclusions Supplementary to the previous GWAS, our study provide an additional evidence on the prognostic value of $9 q 34.2$ rs633862 variant in EOC patients, and this variant may function by regulating the $A B O$ mRNA expression. 


\section{Can 9q34.2 rs633862 polymorphism predicate survival in epithelial ovarian}

2 cancer?

4 Rong JIANG, MD ${ }^{1, \S}$; Yuan XU, MD ${ }^{2, \S}$; Pan WANG, MD ${ }^{1, \S}$; Xi CHENG, MD, PhD 3 ; Tingyan

$5 \mathrm{SHI}, \mathrm{MD}, \mathrm{PhD}^{1,2 *}$; Rongyu $\mathrm{ZANG}, \mathrm{MD}, \mathrm{PhD}^{1,3 *}$

6 Authors' affiliations

$7 \quad{ }^{1}$ Ovarian Cancer Program, Division of Gynecologic Oncology, Department of Obstetrics and

8 Gynecology, Zhongshan hospital, Fudan University, Shanghai, China

$9 \quad{ }^{2}$ Cancer Institute, Fudan University Shanghai Cancer Center, Shanghai, China

$10{ }^{3}$ Gynecologic Oncology, Fudan University Shanghai Cancer Center, Shanghai, China

$\S$ These authors contributed equally to this work.

\section{*Corresponding author}

Tingyan SHI, Ovarian Cancer Program, Division of Gynecologic Oncology, Department of Obstetrics and Gynecology, Zhongshan Hospital, Fudan University, 180 Fenglin Road, Shanghai 200032, China, Tel: +86 21 64041990, Email: tyshi80@163.com Rongyu ZANG, Ovarian Cancer Program, Division of Gynecologic Oncology, Department of Obstetrics and Gynecology, Zhongshan Hospital, Fudan University, 180 Fenglin Road, Shanghai 200032, China, Tel: +86 21 60267406, Email: zang.rongyu@zs-hospital.sh.cn

Short title $A B O$ variant and ovarian cancer prognosis 


\section{Abstract}

22 Objective Our previous genome-wide association study (GWAS) has identified that the $A B O$

23 rs633862 variant in Chromosome 9q34.2 was associated with the risk of epithelial ovarian cancer

24 (EOC) in Chinese Han women. The aim of present study was to evaluate its prognostic effect on

25 EOC.

26 Methods A total of 669 EOC patients were enrolled for the genotyping of rs633862 variant in 9q34.2. We used Kaplan-Meier survival curves, univariate and multivariate Cox proportional hazard models to evaluate the association of rs633862 with overall survival (OS) in EOC patients.

Results We found that rs633862 variant AG/GG genotypes were significantly associated with a longer OS by using univariate Cox proportional hazards regression analysis, compared with the rs633862 AA genotype $(\mathrm{HR}=0.69,95 \% \mathrm{CI} 0.49-0.98, p=0.035)$, albeit with a boardline significance in the multivariate analysis. Similar findings were observed in the subgroup of highgrade serous ovarian carcinoma. Further expression quantitative trait loci (eQTL) analysis indicated that the rs633862 AA genotype was associated with an increased level of $A B O$ mRNA expression $\left(p=1.8 \times 10^{-11}\right)$.

Conclusions Supplementary to the previous GWAS, our study provide an additional evidence on the prognostic value of 9q34.2 rs633862 variant in EOC patients, and this variant may function by regulating the $A B O$ mRNA expression. 
Introduction

42 Ovarian cancer is a common gynecologic malignancy, leading to the fifth cause of cancer death

43 among women worldwide (Torre et al. 2015). In China, there were approximately 52,100 newly

44 diagnosed cases and 22,500 deaths from ovarian cancer in 2015 (Chen et al. 2016). To date, the

45 majority of epithelial ovarian cancer (EOC) patients are treated with primary debulking surgery

46 followed by platinum/taxane chemotherapy, and show a good initial response. However, the

47 five-year overall survival rate remains poor, nearly $30 \%-39 \%$ for advanced stage disease

48 (Pfisterer et al. 2006). Both clinicopathologic and genetic factors contribute to the mortality of

49 EOC patients. In recent decades, genetic variants were found to play an important role in the

50 development and progression of cancers, of which single nucleotide polymorphism (SNP) is the

51 most commonly type that could influence patients' prognosis (Li et al. 2013). Previous studies

52 have identified several gene loci associated with clinical outcomes of EOC by using genetic

53 association studies (Juan et al. 2016) (Bolton et al. 2012), but the underlying mechanism remains

54 unclear.

$55 A B O$ (ABO blood group) gene, located on chromosome 9q34, is well-known to determine

blood type. The encoded glycosyltransferases catalyze the transfer of nucleotide donor sugars to

57 the $\mathrm{H}$ antigen and thus form the ABO blood group antigens (Yazer 2005). Common variants lead

58 to various glycosyltransferases and oligosaccharide antigens, and eventually cause four

59 phenotypes: N-acetylgalactosamine for blood type A, D-galactose for blood type B, both for

60 blood type $\mathrm{AB}$, and neither for an unmodified $\mathrm{H}$ antigen in blood type O. Accumulated data have

61 shown that the genetic diversity of the $A B O$ loci may be caused by multiple alleles, various 
62

mutations and frequent recombination events (Hamosh et al. 2002, Yamamoto et al. 2012). ABO blood group has been demonstrated to be associated with clinical outcomes of pancreatic cancer (Rahbari et al. 2012), esophageal squamous cell cancer (Sun et al. 2014), colon cancer (Cao et al. 2014), nasopharyngeal cancer (Ouyang et al. 2013), and breast cancer (Cihan 2014).

Recently, Cozzi et al reported that blood type A was significantly associated with a longer survival in Caucasian ovarian cancer patients (Cozzi et al. 2017). However, in Chinese ethnics, the result was opposite (NingLi et al. 2015). In addition, to our knowledge, there was no report on the association between genetic variations in the $A B O$ gene and ovarian cancer survival. Our previous three-stage genome-wide association study (GWAS) has identified an EOC susceptibility-associated locus (rs633862) in the $A B O$ gene on chromosome 9q34.2 (Chen et al. 2014). In 2015, a pooled analysis from the Ovarian Cancer Association Consortium (OCAC) and the Consortium of Investigators of Modifiers of $B R C A 1 / 2$ (CIMBA), also validated the role of 9q34.2 loci in EOC risk estimate. Rs633862, on chromosome 9q34.2, is located at 5kb upstream of the $A B O$ gene, and has a minor A allele frequency of 0.456 in Chinese Han population. However, its roles on ovarian cancer survival has not been well illustrated.

The present study aimed to identify the potential function of 9q34.2 rs633862 in EOC patients, hypothesizing that the rs633862 variant was associated with ovarian cancer survival. Based on the results of the previous three-stage GWAS (Chen et al. 2014), we here conducted a retrospective analysis among 669 Han Chinese patients with EOC.

\section{Materials and methods}


83

84

85

86

87

88

\section{Ethics Statement}

The study was approved by the Institutional Review Board of Fudan University Cancer Center (FUSCC) (Ethical Application Ref: 050432-4-1212B). A written informed consent was obtained from all recruited individuals.

\section{Study subjects}

The patients were collected by Shanghai Ovarian Cancer Study as described previously in the Chinese EOC GWAS, mainly from FUSCC between 2009 to 2012 as described previously (Shi et al. 2015). All patients were unrelated Han Chinese from eastern China, and histopathologically confirmed independently as primary EOC by gynecologic pathologists as routine diagnosis. As a result, a cohort of 699 patients with both GWAS genotyping data and available follow-up data were included into the analysis. The detailed clinical and pathological information of patients were extracted from the patients' electronic database at FUSCC, included FIGO (International Federation of Gynecology and Obstetrics, 2009) stage, histopathology, tumor grade, pelvic lymph node (LN) metastasis, the expression of estrogen receptor (ER) and progesterone receptor (PR), chemotherapy schemes and residual disease after primary cytoreduction. For patients who received adjuvant chemotherapy with platinum and paclitaxel for six to eight cycles, follow-up was conducted in out-patient clinics or by telephone calls. Patients were followed every six months. If the patients could not be followed up for continuous three times, they will be considered as unavailable follow-up data and be excluded in the current study. Overall survival (OS) was defined as the time from the initiation of therapy (primary debulking surgery or first 
104

105

106

107

108

109

110

111

112

113

114

115

116

117

118

119

120

121

122

123

124

cycle of neoadjuvant chemotherapy) to the date of the last follow-up or the date to death of ovarian cancer. Patients without progression were censored at the date of last record.

\section{Genotyping}

An additional $10 \mathrm{ml}$ of venous blood sample (collected by the Tissue Bank of FUSCC after the diagnosis and before the initiation of treatment) were kept frozen till DNA extraction for genotyping. According to the standard protocol, genomic DNA was extracted from peripheral blood samples. Genotyping was performed using iPLEX MassARRAY platform (Sequenom, Inc) as reported previously (Chen et al. 2014). Five percent of the samples were randomly selected for repeated genotyping, and the results were $100 \%$ concordant.

\section{Statistical analysis}

We used Kaplan-Meier survival curves to visualize the OS by genotyping, and log-rank test for differences in the survival times among patients. Univariate and multivariate Cox proportional hazards models were used to evaluate the effects of genotypes and clinicopathological variables on patients' OS by computing hazard ratios (HRs) and their 95\% confidence intervals (CIs).

Multivariate analyses were adjusted by those variables that were independently associated with survival in the univariate model. All of those tests were two-sided with a statistical significance set at $p<0.05$, and were performed using SAS 9.1 software (SAS Institute, Cary, NC). 
125 We explored the function consequences of 9q34.2 (rs633862) using two in silico tools, including

126 SNPinfo (http://snpinfo.niehs.nih.gov/snpfunc.htm) and UCSC Genome Browser

127 (http://genome.ucsc.edu) (Kuhn et al. 2007). To evaluate the association between rs633862 and

128 gene expression in EOC, the publicly GTExPortal dataset (http://gtexportal.org) was used for

129 expression quantitative trait loci (e QTL) analysis.

130

131

\section{Results}

132

133

134

135

136

137

138

139

140

141

142

143

144

145

\section{Associations between genotypes and overall survival}

The detailed clinical and pathological information was listed in Table 1. Out of 669 EOC

patients, $136(20.3 \%)$ died at the last follow-up time (June, 2014), with a median overall survival of 31.5 months. As shown in Figure 1, patients who carried rs633862 AG/GG genotypes had a longer OS, compared with AA genotype carriers (log-rank test, $p=0.035$ ). Further univariate Cox proportional hazards regression analyses confirmed our data that rs633862 was significantly associated with EOC prognosis (AG/GG genotypes, $\mathrm{HR}=0.69,95 \% \mathrm{CI} 0.49-0.98, p=0.035$ ), albeit with a boardline significance in multivariate analysis $(p=0.103)$ (Table 2$)$. Interestingly, the similar prognostic value of rs633862 in overall survival was illustrated in the subgroup of high-grade serous ovarian carcinoma (univariate model: $\mathrm{HR}=0.67,95 \% \mathrm{CI} 0.46-0.99, p=0.046$;

Table 3). Moreover, age, FIGO staging, histological type and complete cytoreductive surgery were significantly associated with overall survival independently ( $p<0.05$ for all, Table 2$)$. In the multivariate Cox proportional hazards model, age above 54.5 years old (adjusted HR=1.04, 95\%CI 1.01-1.06), FIGO stage III-IV (adjusted HR=8.09, 95\%CI 1.07-61.26) and complete 
146

147 (Table 2).

148

149

150

151

152

153

154

155

156

157

158

159

160

161

162

163

164

165

166 EOC patients.

\section{Discussion}

cytoreductive surgery (adjusted $\mathrm{HR}=0.39,95 \% \mathrm{CI}$ 0.21-0.71) remained statistically significant

\section{Bioinformatics prediction between $9 q 34.2$ rs633862 and ovarian cancer}

We used the SNPinfo online tool to evaluate plausible biological mechanisms underlying the observed association, and found that 9q34.2 rs633862 was located at 4814 bp upstream of the $A B O$ gene, and predicted as a transcription factor binding site (TFBS) (Supplementary Table

S1). By using the public GTExPortal database, we evaluated the available $A B O \mathrm{mRNA}$ expression in relation to rs633862 genotypes in a total of $17 \mathrm{GG}, 37 \mathrm{AG}$, and $31 \mathrm{AA}$ carriers.

According to the eQTL information analysis (Figure 2), rs633862 AA genotype was associated with an increased level of $A B O$ mRNA expression $\left(p=1.8 \times 10^{-11}\right)$. These findings indicated that the rs633862 $\mathrm{G} \rightarrow \mathrm{A}$ variant may regulate $A B O$ expression, leading to the increased mortality of

In this study, we investigated the associations between a potentially functional variant in chr9q34.2 (rs633862) and overall survival of EOC patients. Our data showed that patients carrying rs633862 AG/GG genotypes had a longer OS, compared with those with AA genotype. Furthermore, genotype-phenotype correlation analyses suggested that rs633862 involved in regulating $A B O$ mRNA expression, indicating its potential function on modulating ovarian cancer progression. 
167 Previous studies have demonstrated the association between ABO blood group antigens and 168 clinical outcome in several human cancers, such as esophageal squamous cell cancer (Shiratori et

169

170

171

172

173

174 175

176

177

178

179

180

181

182

183

184

185

186

187

al. 2016), laryngeal cancer (Ting et al. 2016), gastric cancer (Xu et al. 2016) (Angelov et al.

1900) and non-small cell lung cancer (NingLi et al. 2015). Also, the association between ABO

blood group and ovarian cancer was reported, but the results were opposite between Caucasians

and Chinese (Cozzi et al. 2017, (NingLi et al. 2015). Moreover, the mechanisms of ABO blood

group on malignancies were still unclear. $A B O$ gene encodes glycotransferases, which is a

crucial catalyst in the formation of $\mathrm{ABO}$ antigens. The expression of $\mathrm{ABO}$ antigen is not only on

blood cell membranes, but also on the surface epithelium and inclusion cysts of the ovary

(Welshinger et al. 1996). Blood group antigens expressed on tumor cells could influence

tumorigenesis, cell motility and immune escape (Pendu et al. 2001). In addition, recent studies

have reported that $A B O$ types were associated with levels of soluble ICAM-1 (Paré et al. 2008),

tumor necrosis factor-alpha (Melzer et al. 2008), and soluble E-selection (Qi et al. 2010),

indicating the possible role of blood group antigens in immune response.

To the best of our knowledge, this is the first study demonstrating the predictive value of $A B O$

variants in EOC among Han Chinese women. Interestingly, we found that the rs633862 AG/GG

genotypes might be a protective factor for overall survival. Further bioinformatics analyses

showed that the genomic region containing 9q34.2 (rs633862), located in the upstream of $A B O$

gene, was predicted as a transcription factor binding site, and could affect the biosynthesis of

protein. Genotype-phenotype correlation analysis from the online GTExPortal database indicated

a significant association of $A B O$ mRNA expression levels with rs633862 genotypes. Therefore, 
we concluded that rs633862 AA genotype may function by up-regulating $A B O$ mRNA expression and thus contribute to a worse OS in Chinese EOC patients.

Several limitations in the present retrospective study need to be addressed. First, the sample size and the follow-up time seems relatively limited, which might be a main reason for the boardline significance of rs633862 in the multivariate model analysis. Second, we used bioinformatics to predict the potential function of $A B O$ gene, further validation of genotypephenotype correlation by using the current study samples and functional experiments focused on rs633862 variant are needed to illustrate the potential mechanisms. Third, further studies should include more polymorphisms in the $A B O$ gene and related regions.

In summary, as the supplementary of the previous GWAS, our study provides an additional evidence of 9q34.2 rs633862 variant on ovarian cancer prognosis. Rs633862 AA genotype may function by up-regulating $A B O$ mRNA expression, and thus contribute to clinical outcomes in Chinese ovarian cancer patients. However, well-designed larger, prospective studies with longer follow-up time and further functional analyses are warranted to validate our findings.

\section{Acknowledgements}

We would like to thank Qingyi Wei from Duke Cancer Institute, as well as Kexin Chen and Lian

Li from Tianjin Medical University Cancer Hospital, for their supports on genotyping from Chinese EOC genome-wide association study. We also thank Ruoxin Zhang from Fudan University Shanghai Cancer Center for her help on bioinformatics analysis and reviewing this 
209 manuscript.

210 


\section{Figure legends}

212 Figure1. Kaplan-Meier estimates for the survival of patents according to rs633862 genotypes.

213 Rs633862 variant AG/GG genotype carriers were shown a significantly longer overall survival, 214 compared with AA genotype carriers ( $\mathrm{HR}=0.69,95 \% \mathrm{CI} 0.49-0.98, p=0.035)$.

Figure 2. $A B O$ mRNA expression by eQTL analysis in patients with EOC. The rs633862 AA genotype was associated with an increased level of $A B O$ mRNA expression $(p<0.05)$. Homo Ref, rs633862 GG genotype; Het, rs633862 AG genotype; Homo Alt, rs633862 AA genotype. 
220

221

222

223

224

225

226

227

228

229

230

231

232

233

234

235

236

237

238

239

240

\section{References}

Angelov KG, Vasileva MB, Grozdev KS, Sokolov MB, and Todorov G. 2014. Clinical and pathological characteristics, and prognostic factors for gastric cancer survival in 155 patients in Bulgaria. Hepato-gastroenterology 61:2421-2424.

Bolton KL, Ganda C, Berchuck A, Pharaoh PD, and Gayther SA. 2012. Role of common genetic variants in ovarian cancer susceptibility and outcome: progress to date from the Ovarian Cancer Association Consortium (OCAC). Journal of Internal Medicine 271:366-378.

Cao X, Wen ZS, Sun YJ, Li Y, Zhang L, and Han YJ. 2014. Prognostic value of ABO blood group in patients with surgically resected colon cancer. British Journal of Cancer 111:174-180.

Chen K, Ma H, Li L, Zang R, Wang C, Song F, Shi T, Yu D, Yang M, Xue W, Dai J, Li S, Zheng H, Wu C, Zhang Y, Wu X, Li D, Xue F, Li H, Jiang Z, Liu J, Liu Y, Li P, Tan W, Han J, Jie J, Hao Q, Hu Z, Lin D, Ma D, Jia W, Shen H, and Wei Q. 2014. Genome-wide association study identifies new susceptibility loci for epithelial ovarian cancer in Han Chinese women. Nat Commun 5:4682.

Chen W, Zheng R, Baade PD, Zhang S, Zeng H, Bray F, Jemal A, Yu XQ, and He J. 2016. Cancer statistics in China, 2015. Ca A Cancer Journal for Clinicians 66:115.

Cihan YB. 2014. Significance of ABO-Rh blood groups in response and prognosis in breast cancer patients treated with radiotherapy and chemotherapy. Asian Pac J Cancer Prev 15:40554060.

Cozzi GD, Levinson RT, Toole H, Snyder MR, Deng A, Crispens MA, Khabele D, and BeeghlyFadiel A. 2017. Blood type, ABO genetic variants, and ovarian cancer survival. PLoS One 
242

Hamosh A, Scott AF, Amberger J, Bocchini C, Valle D, and McKusick VA. 2002. Online Mendelian Inheritance in Man (OMIM), a knowledgebase of human genes and genetic disorders. Nucleic Acids Res 30:52-55.

Juan W, Shan K, Na W, Rong-Miao Z, and Yan L. 2016. The Associations of Genetic Variants in E-cadherin Gene With Clinical Outcome of Epithelial Ovarian Cancer. International Journal of Gynecological Cancer 26:1601-1607.

Kuhn RM, Karolchik D, Zweig AS, Trumbower H, Thomas DJ, Thakkapallayil A, Sugnet CW, Stanke M, Smith KE, and Siepel A. 2007. The UCSC genome browser database: update 2007. Nucleic Acids Research 35:D668.

Li C, Yin M, Wang LE, Amos CI, Zhu D, Lee JE, Gershenwald JE, Grimm EA, and Wei Q. 2013. Polymorphisms of Nucleotide Excision Repair Genes Predict Melanoma Survival. Journal of Investigative Dermatology 133:1813.

Melzer D, Perry JRB, Hernandez D, Corsi AM, Stevens K, Rafferty I, Lauretani F, Murray A, Gibbs JR, and Paolisso G. 2008. A Genome-Wide Association Study Identifies Protein Quantitative Trait Loci (pQTLs). Plos Genetics 4:e1000072.

NingLi, MiaoXu, ChaoFengLi, WeiOu, BaoXiaoWang, SongLiangZhang, PengFeiXu, ChengYuan, QunAiHuang, and SiYuWang. 2015. Prognostic role ofthe ABO blood types inChinese patients withcuratively resected non-small cell lung cancer:a retrospective analysis of1601 cases ata single cancer center. Chinese Journal of Cancer 34:475-482.

Ouyang PY, Su Z, Mao YP, Liu Q, and Xie FY. 2013. Prognostic value of ABO blood group in 

Cancer 109:2462-2466.

264

265

266

267

268

269

270

271

272

273

274

275

276

277

278

279

280

281

282

Paré G, Chasman DI, Kellogg M, Zee RYL, Rifai N, Badola S, Miletich JP, and Ridker PM. 2008.

Novel Association of ABO Histo-Blood Group Antigen with Soluble ICAM-1: Results of a Genome-Wide Association Study of 6,578 Women. Plos Genetics 4:e1000118.

Pendu JL, Marionneau S, Cailleau-Thomas A, Rocher J, Moullac-Vaidye BL, and Clément M. 2001. ABH and Lewis histo-blood group antigens in cancer. Apmis Acta Pathologica Microbiologica Et Immunologica Scandinavica 109:9.

Pfisterer J, Plante M, Vergote I, Du BA, Hirte H, Lacave AJ, Wagner U, Stähle A, Stuart G, and Kimmig R. 2006. Gemcitabine plus carboplatin compared with carboplatin in patients with platinum-sensitive recurrent ovarian cancer: an intergroup trial of the AGO-OVAR, the NCIC CTG, and the EORTC GCG. Journal of Clinical Oncology Official Journal of the American Society of Clinical Oncology 24:4699.

Qi L, Cornelis MC, Kraft P, Jensen M, van Dam RM, Sun Q, Girman CJ, Laurie CC, Mirel DB, and Hunter DJ. 2010. Genetic variants in ABO blood group region, plasma soluble Eselectin levels and risk of type 2 diabetes. Human Molecular Genetics 19:1856.

Rahbari NN, Bork U, Hinz U, Leo A, Kirchberg J, Koch M, Büchler MW, and Weitz J. 2012. AB0 blood group and prognosis in patients with pancreatic cancer. BMC cancer 12:319.

Shi TY, Jiang Z, Jiang R, Yin S, Wang MY, Yu KD, Shao ZM, Sun MH, Zang R, and Wei Q. 2015. Polymorphisms in the kinesin-like factor $1 \mathrm{~B}$ gene and risk of epithelial ovarian cancer in Eastern Chinese women. Tumor Biology 36:6919-6927. 
283 Shiratori F, Shimada H, Yajima S, Suzuki T, Oshima Y, Nanami T, Ito M, and Kaneko H. 2016.

284

285

286

287

288

289

290

291

292

293

294

295

296

297

298

299

300

301

302

303
Relationship between ABO blood group and clinicopathological factors and their effect on the survival of Japanese patients with esophageal squamous cell carcinoma. Surgery Today:1-7.

Sun P, Chen C, Zhang F, An X, Li XY, Li YH, Zhu ZH, and Wang FH. 2014. The ABO blood group predicts survival in esophageal squamous cell carcinoma in patients who ever smoked: a retrospective study from China. Tumor Biology 35:7201-7208.

Ting, Pei-Jing, Xiao-Zhong, Chen, and Wei-Han. 2016. ABO blood group is a predictor of survival in patients with laryngeal cancer. Chinese Journal of Cancer 35:706-712.

Torre LA, Bray F, Siegel RL, Ferlay J, Lortet-Tieulent J, and Jemal A. 2015. Global cancer statistics, 2012. CA Cancer J Clin 65:87.

Welshinger M, Finstad CL, Venkatraman E, Federici MG, Rubin SC, Jr LJ, and Lloyd KO. 1996. Expression of $\mathrm{A}, \mathrm{B}$, and $\mathrm{H}$ blood group antigens in epithelial ovarian cancer: relationship to tumor grade and patient survival. Gynecol Oncol 62:106-112.

Xu YQ, Jiang TW, Cui YH, Zhao YL, and Qiu LQ. 2016. Prognostic value of ABO blood group in patients with gastric cancer. Journal of Surgical Research 201:188.

Yamamoto F, Cid E, Yamamoto M, and Blancher A. 2012. ABO research in the modern era of genomics. Transfus Med Rev 26:103-118.

Yazer MH. 2005. What a difference 2 nucleotides make: a short review of ABO genetics. Transfusion Medicine Reviews 19:200-209. 


\section{Table 1 (on next page)}

Table 1

Clinical characteristics of epithelial ovarian cancer patients. 
1 Table 1

2 Clinical characteristics of epithelial ovarian cancer patients

\begin{tabular}{|c|c|c|}
\hline \multirow[t]{2}{*}{ Characteristics } & \multicolumn{2}{|c|}{ Patients } \\
\hline & $\mathrm{N}=669$ & $\%$ \\
\hline Age, (mean $\pm S D)$, yrs & $54.3 \pm 0.39$ & \\
\hline \multicolumn{3}{|l|}{ FIGO stage } \\
\hline I & 15 & 3.9 \\
\hline II & 27 & 7.0 \\
\hline III & 293 & 76.3 \\
\hline IV & 49 & 12.8 \\
\hline \multicolumn{3}{|l|}{ Histopathology } \\
\hline High-grade Serous & 476 & 71.3 \\
\hline Low-grade Serous & 76 & 11.4 \\
\hline Endometrioid & 38 & 5.7 \\
\hline Clear Cell & 32 & 4.8 \\
\hline Mucinous & 22 & 3.3 \\
\hline Others & 23 & 3.5 \\
\hline \multicolumn{3}{|l|}{ Tumor grade } \\
\hline Grade 1 & 11 & 1.9 \\
\hline Grade 2 & 100 & 16.9 \\
\hline Grade 3 & 481 & 81.3 \\
\hline \multicolumn{3}{|l|}{ Pelvic LN metastasis } \\
\hline Negative & 175 & 53.0 \\
\hline Positive & 155 & 47.0 \\
\hline \multicolumn{3}{|l|}{ ER expression } \\
\hline Negative & 137 & 30.3 \\
\hline Positive & 315 & 69.7 \\
\hline \multicolumn{3}{|l|}{ PR expression } \\
\hline Negative & 290 & 63.2 \\
\hline Positive & 169 & 36.8 \\
\hline \multicolumn{3}{|c|}{ Residual disease after primary cytoreduction } \\
\hline 0 ( no grossly visible tumor) & 221 & 35.3 \\
\hline
\end{tabular}




$\begin{array}{lcccc}3 & 1(0.1-0.5 \mathrm{~cm}) & 113 & 18.0 & \text { FIGO, } \\ 4 & 2(0.5-1.0 \mathrm{~cm}) & 144 & 23.0 & \text { International } \\ 5 & 3(>1.0 \mathrm{~cm}) & 149 & 23.8 & \text { Federation of } \\ 6 & \text { Recurrence } & & & \text { Gynecology } \\ 7 & \text { No } & 173 & 25.9 & \text { and } \\ 8 & \text { Yes } & 496 & 74.1 & \text { Obstetrics; } \\ 9 & \text { Death } & & & \text { LN, lymph } \\ 10 & \text { No } & 553 & 79.7 & \text { node; ER, } \\ 11 & \text { Yes } & 136 & 20.3 & \text { estrogen } \\ 12 & & & & \text { receptor; PR, } \\ 13 & \text { progesterone receptor. } & & & \end{array}$




\section{Table 2 (on next page)}

Table 2

Associations of variables with the survival of epithelial ovarian cancer by Univariate and Multivariate Cox proportional hazards models. 
1 Table 2. Associations of variables with the survival of epithelial ovarian cancer by Univariate 2 and Multivariate Cox proportional hazards models.

3

Variables

\begin{tabular}{cccccc} 
No. & No. of death & \multicolumn{2}{c}{ Univariate model } & \multicolumn{2}{c}{ Multivariate model } \\
\cline { 2 - 6 } of & $(\%)$ & HR $(95 \%$ & $P$ & HR (95\% CI)* & $P *$ \\
cases & & CI) & & & \\
& & & & & \\
\end{tabular}

\section{All}

$669 \quad 136(20.3)$

Age, yrs

$\begin{array}{ccccccc}>54.5(\text { median }) & 332 & 77(23.2) & 1.61(1.15- & \mathbf{0 . 0 0 6} & 1.04(1.01-1.06) & \mathbf{0 . 0 0 2} \\ 2.27) & & \end{array}$

FIGO stage

\begin{tabular}{|c|c|c|c|c|c|}
\hline III-IV & 342 & $90(26.3)$ & $\begin{array}{c}17.85(2.48- \\
128.76)\end{array}$ & 0.004 & $\begin{array}{c}8.09(1.07- \\
61.26)\end{array}$ \\
\hline
\end{tabular}

Histopathology

\begin{tabular}{|c|c|c|c|c|c|}
\hline HGSOC & 476 & 103 (21.6) & $\begin{array}{c}1.60(1.07- \\
2.38)\end{array}$ & 0.022 & $\begin{array}{c}1.12(0.60- \\
2.08)\end{array}$ \\
\hline
\end{tabular}

\section{Complete cytoreduction}

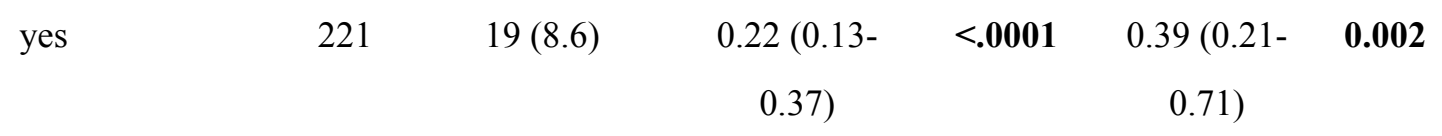

\section{Rs633862 genotype}

\begin{tabular}{|c|c|c|c|c|c|}
\hline AG/GG & 438 & 77 (56.6) & $\begin{array}{c}0.69(0.49- \\
0.98)\end{array}$ & 0.035 & $0.70(0.46-1.08)$ \\
\hline
\end{tabular}

4 HGSOC, high grade serous ovarian carcinoma.

$5 *$ Multivariate analyses were adjusted by those variables that were independently associated with

6 survival in the univariate model, in this case, including age, FIGO stage, histopathology and

7 complete cytoreduction. 


\section{Table 3 (on next page)}

Table 3

Prognostic value of rs633862 AG/GG genotypes in the subgroup of histopathology. 
1 Table 3. Prognostic value of rs633862 AG/GG genotypes in the subgroup of histopathology.

2

Variables

\begin{tabular}{cccccc} 
No. & No. of death & \multicolumn{2}{c}{ Univariate model } & \multicolumn{2}{c}{ Multivariate model } \\
\cline { 3 - 6 } of & $(\%)$ & HR $(\mathbf{9 5} \%$ & $P$ & HR $(\mathbf{9 5 \%}$ CI) & $P$ \\
cases & & CI) & & & \\
& & & & &
\end{tabular}

\section{Histopathology}

\begin{tabular}{|c|c|c|c|c|c|c|}
\hline HGSOC & 311 & $56(18.0)$ & $\begin{array}{c}0.67(0.46- \\
0.99)\end{array}$ & 0.046 & $\begin{array}{c}0.65(0.41- \\
1.03)\end{array}$ & 0.064 \\
\hline Non-HGSOC & 126 & $21(16.7)$ & $\begin{array}{c}0.80(0.39- \\
1.65)\end{array}$ & 0.550 & $\begin{array}{c}1.01(0.29- \\
3.53)\end{array}$ & 0.990 \\
\hline Low-grade Serous & 53 & $11(20.8)$ & $\begin{array}{c}0.69(0.25- \\
1.91)\end{array}$ & 0.476 & $\begin{array}{c}0.32(0.07- \\
1.45)\end{array}$ & 0.138 \\
\hline Endometrioid & 22 & $2(9.1)$ & $\begin{array}{c}0.76(0.11- \\
5.37)\end{array}$ & 0.780 & - & 0.999 \\
\hline Clear Cell & 22 & $2(9.1)$ & $\begin{array}{c}0.94(0.09- \\
10.39)\end{array}$ & 0.959 & - & 1.000 \\
\hline Mucinous & 14 & $3(21.4)$ & $\begin{array}{c}0.44(0.09- \\
2.21)\end{array}$ & 0.317 & - - & 1.000 \\
\hline
\end{tabular}


Figure 1

Figure 1. Kaplan-Meier estimates for the survival of patents according to rs633862 genoty pes.

Rs633862 variant AG/GG genotype carriers was shown a significantly longer overall survival, compared with rs633862 AA genotype carriers ( $\mathrm{HR}=0.69,95 \% \mathrm{Cl} 0.49-0.98, p=0.035)$.

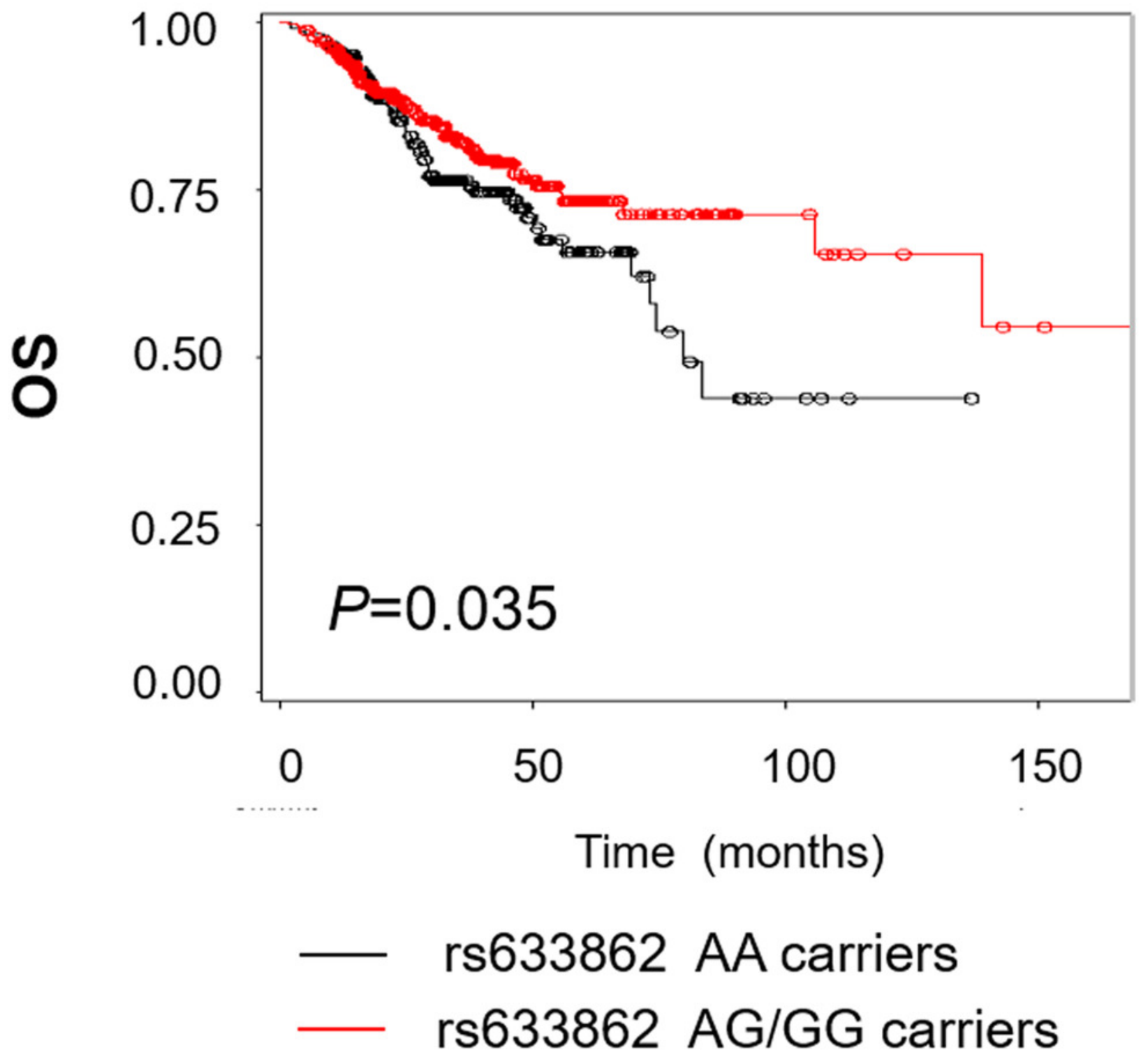


Figure 2

Figure 2. $A B O$ mRNA expression by eQTL analysis in patients with EOC.

Figure 2. $A B O$ mRNA expression by eQTL analysis in patients with EOC. The rs633862 AA genotype was associated with an increased level of $A B O$ mRNA expression ( $p<0.05$ ). Homo Ref, rs633862 GG genotype; Het, rs633862 AG genotype; Homo Alt, rs633862 AA genotype.

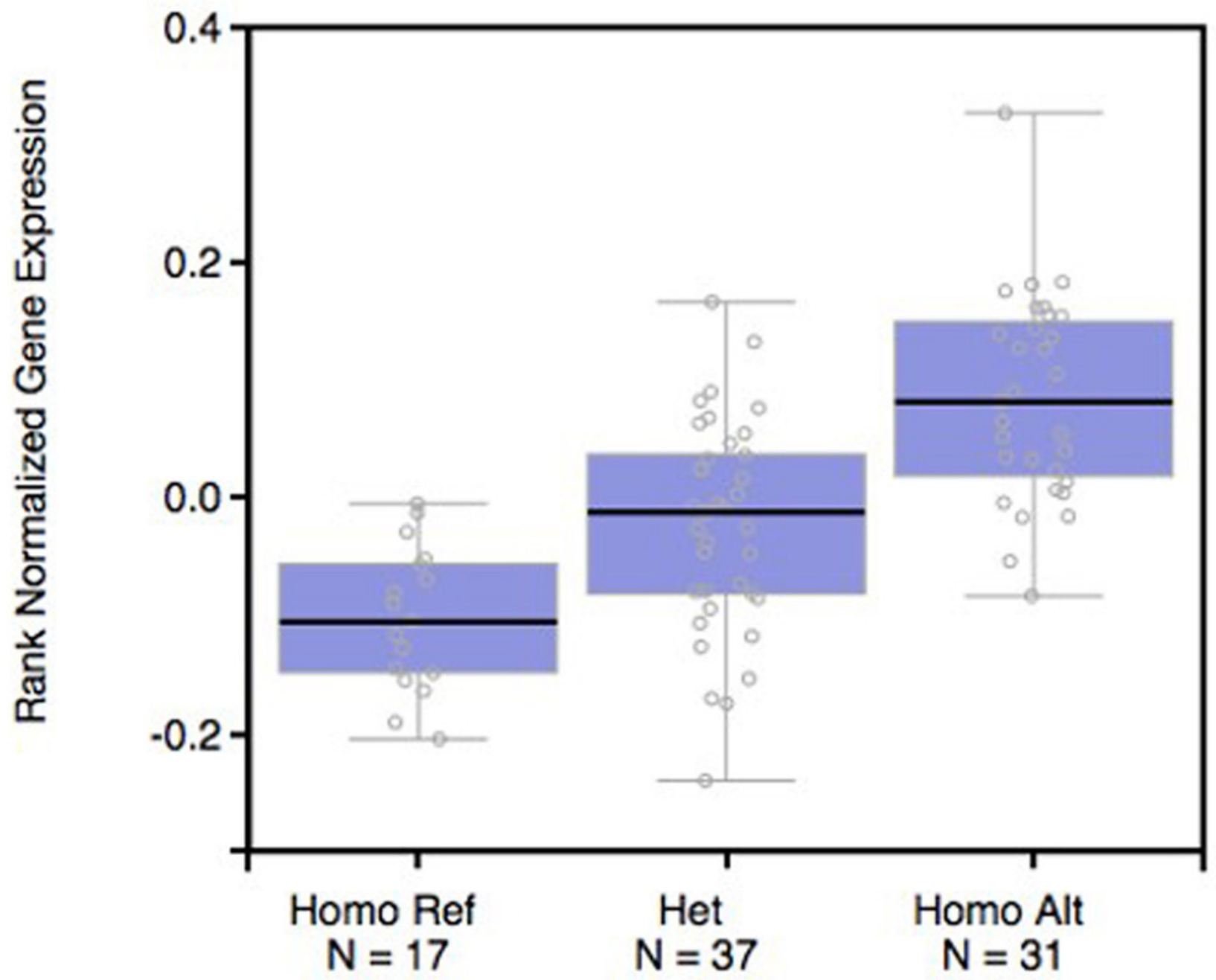

